\title{
Infant Reflux and Aerophagia Associated with the Maxillary Lip-tie ${ }^{1}$ and Ankyloglossia (Tongue-tie)
}

Lawrence Kotlow, DDS 2

\begin{abstract}
Successful breastfeeding depends on an infant's latching onto the mother's breast correctly. If an excessive amount of air reaches the stomach due to a poor latch, abdominal distension, belching, and flatulence often develop. This may be the result of pathologic aerophagia. Aerophagia (from the Greek word aerophagein: "to eat air") is excessive swallowing of air during feeding. A poor or incorrect latch may be the result of both a maxillary lip-tie and ankyloglossia. The author presents two cases where infants were being treated for reflux by their medical doctors. After revision of both the lip- and tongue-ties, the symptoms were significantly reduced, eliminating the need for medications.
\end{abstract}

Keywords: Ankyloglossia, tongue-tie, lip-tie, reflux, GERD, colic, labial frenulum Clinical Lactation, 2011, Vol. 2-4, 25-29

\section{Introduction}

Successful breastfeeding depends upon a correct latch. For newborns, the chin should rest on the breast below the nipple, the lips should be everted and at least the lower one flanged outward. The jaw is usually open to an angle of 130 to 160 degrees (see http://www.vabarnsley.org.uk/pdf/ breastfeeding/attached_properly.pdf). The baby's top lip should rest just above the mother's nipple, generally leaving the upper portion of the areola exposed. Clicking sounds and nipple pain may indicate inadequate attachment to the breast and/or improper sucking.

When the infant attempts to latch using just the lips to try to maintain a gentle grip on the breast, breastfeeding can be problematic for both the infant and the mother. Problems for the infant may include failure to thrive, prolonged and frequent breastfeeding with poor milk transfer, inability to maintain an effective latch, colic, and reflux. Mothers often develop plugged ducts, pain during feeding, flattened, compressed and injured nipples, and mastitis (Kotlow, 2011; Li et al., 2008)

Consequences of an incorrect latch may include the following: poor suck, poor seal around the breast, loud clicking noises due to tongue recoil, extended feeding times, poor infant weight gain, and infant fussiness and irritability during and after breastfeeding.

A review of the literature reveals no studies concerning the relationship between ankyloglossia, lip-ties, and reflux. Reflux

1 The author has proposed that the term maxillary frenum be redefined as a piece of the upper lip that is attached to the maxillary gingival tissue and therefore is better described as a "lip-tie."

2 kiddsteeth@aol.com and colic symptoms are often discussed in the literature but have not been specifically documented as problems directly related to these oral soft-tissue abnormalities.

Aerophagia, originating from the Greek word aerophagein, which means to "eat air," is the excessive swallowing of air (Chitkara et al., 2005; Courtiol, 2011; Fernando, 1998; Loening-Baucke, 2000). If an excessive amount of air reaches the stomach (pathologic aerophagia), abdominal distension, belching, and flatulence often develop. This may trigger recurrent crying in affected infants.

Colic is a condition that occurs in an otherwise healthy baby. The infant cries or screams frequently and for extended periods, often at the same time of day daily, without any obvious reason. The condition typically appears within the first two weeks of life and may disappear by four to six months of age. Crying, and the exhaustion associated with it, can trigger breastfeeding failure. Mothers are often told that their milk is at fault and are sometimes advised to wean. Similar problems occur in bottle-fed infants.

Reflux or GER (gastroesophageal reflux) in an infant may express itself as irritability and pain, sometimes with constant or sudden crying, or colic-like symptoms. Babies can be inconsolable, especially when laid down flat. Other signs and symptoms of reflux in an infant may include difficulty sleeping when lying down, arching their necks and back during or after feeding, problems swallowing, gagging, coughing, "cud chewing," hiccupping, and drooling. Feeding refusal, or constant feeding, are common responses. Normal movement of the tongue (rippling from the front of the tongue to the back) is difficult or impossible if the tongue is tethered to the floor of the mouth. 
GERD (Gastroesophageal Reflux Disease) is a pathological condition, and a commonly over-diagnosed problem. Infants properly diagnosed as having GERD may show significant signs of failure to thrive and poor growth along with multiple episodes of vomiting. They may suffer from intense pain and refuse to eat due to the pain and discomfort that accompany the reflux. Prior to revision of the soft-tissue abnormalities, parents report the infants often were placed in an infant car carrier due to crying and apparent GI discomfort. After surgery, parents indicate the infants were now able to sleep lying down without discomfort.

Treatment of infants with significant recurring reflux symptoms may often be delayed to see if the problem will resolve itself in three or four months. In severe instances, the infant may be placed on prescription medications, such as Zantac (ranitidine, H2 blockers) or Prevacid (lansoprazole, Proton pump inhibitor), to reduce the reflux symptoms.

\section{The Maxillary Lip-tie (Maxillary Frenum)}

The maxillary frenum is the tissue attaching the upper lip to the infant's gingival tissue (gums). This is a remnant of tissue in the midline of the upper lip and gum (gingiva), which holds the upper lip attached to the gum, and may interfere with the normal mobility and function of the upper lip. Reduced labial range of motion restricts the upward and outward movement of the lip away from the upper gum. The author has developed a clinical classification of infant maxillary lip attachments to assist in the diagnosis and treatment of the maxillary lip-tie (Kotlow, 2004a, 2004b, 2010).

\section{Ankyloglossia (Tongue-tie)}

Ankyloglossia, or tongue-tie, has been defined by the International Affiliation of Tongue-tie Professionals [http:// tongue-tied.net/] as:

The embryologic remnant of the tissue in the midline of the undersurface of the tongue and the floor of the mouth (para 1).

When this remnant of tissue fails to regress by birth, it may interfere with the mobility and function of the tongue. A baby with tongue-tie may be unable to keep the tongue extended for the relatively prolonged job of breastfeeding, and thus the tongue will "recoil" when baby lowers the posterior tongue and jaw during sucking. The clicking sound during breastfeeding indicates that baby is repeatedly breaking the seal or suction. Bottle-fed infants may suffer colic and reflux symptoms as well due to the effects of abnormal maxillary and lingual ties.

Patients who presented to my practice with a history of excessive gas, reflux, and bloating signs and symptoms reported significant relief when the lingual and maxillary ties were revised. The author has treated over 50 infants where revising
Kotlow Classification in Newborns of the Maxillary Lip Attachment: Use for Diagnosis and Evaluation of Lip-tie

A Class 1 lip attachment is often not very observable or prominent, and requires no treatment. This type of attachment is insignificant. A Class 2, 3, or 4 may contribute to breastfeeding problems and should be evaluated for revision. A tight lip attachment often accompanies a short, tight lingual frenum, and is often overlooked.

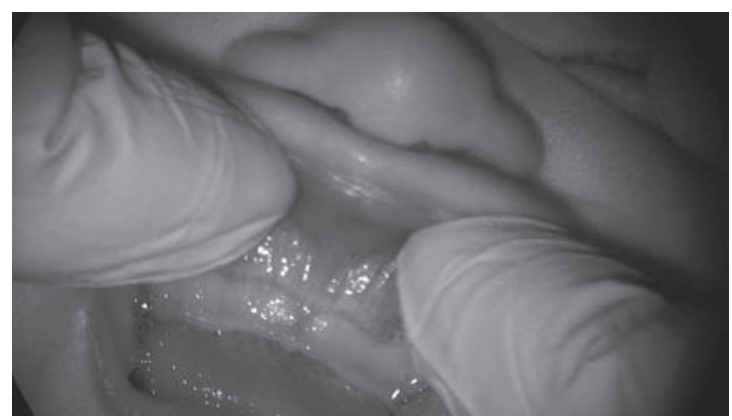

Class 1: Requires no treatment

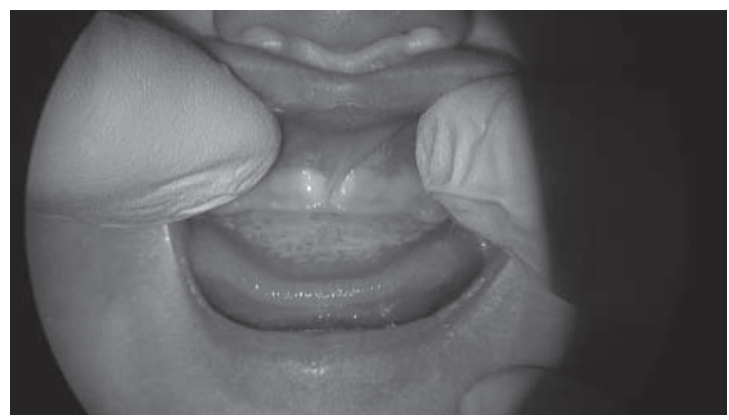

Class 2: Attaching at the junction free and attached gingival tissue

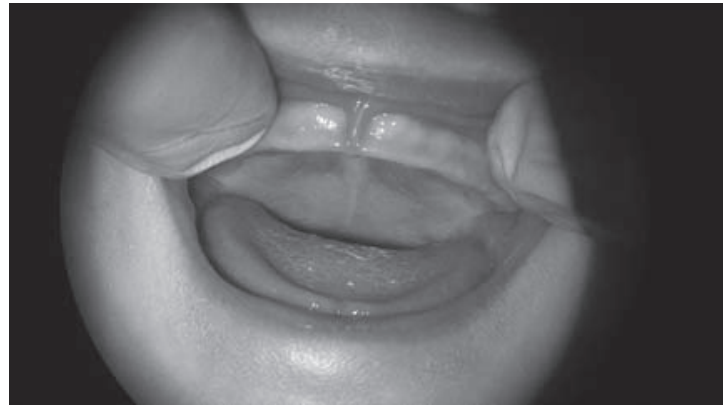

Class 3: Attaching just in front of the anterior papilla

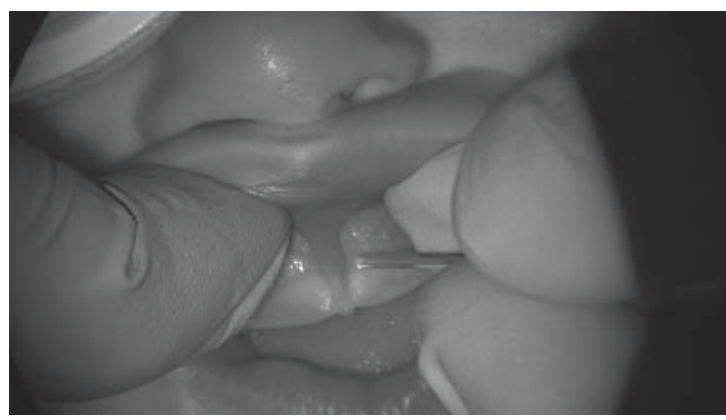

Class 4: Attachment into anterior papilla extending into the hard palate 


\section{Newborn and Infant Tongue-tie Classifications: Use for Diagnosis and Evaluation of Lingual Frenum Attachments}

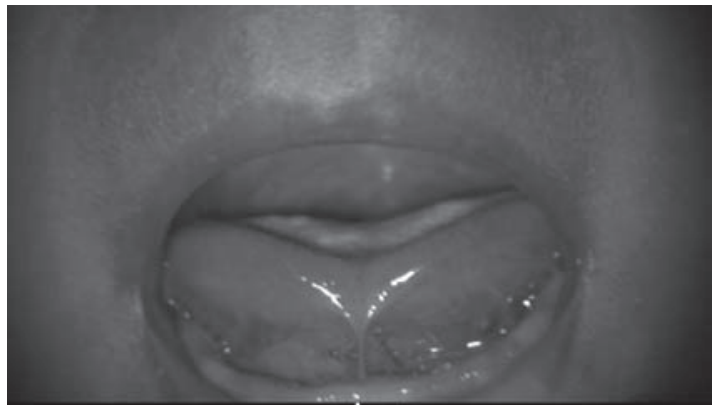

Class 1: 0-3 mm from tip of tongue

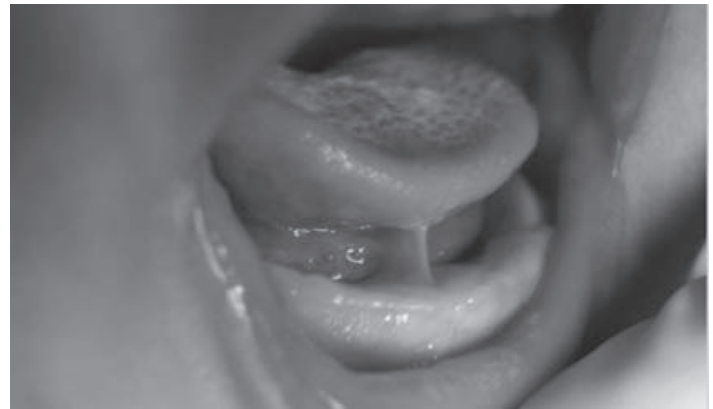

Class 2: 4-6 $\mathrm{mm}$ from tip of tongue

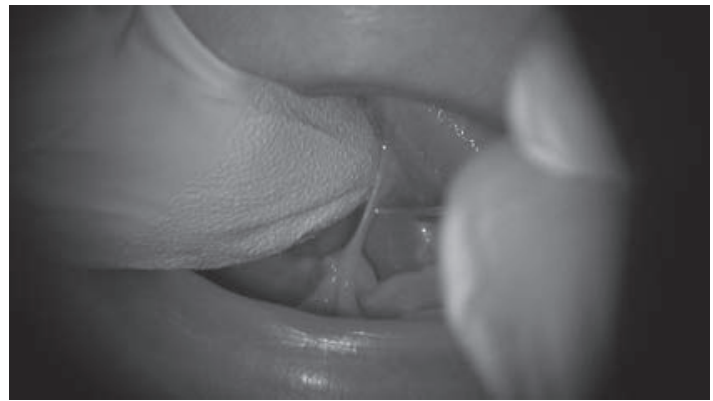

Class 3: 7-9 $\mathrm{mm}$ from tip of tongue

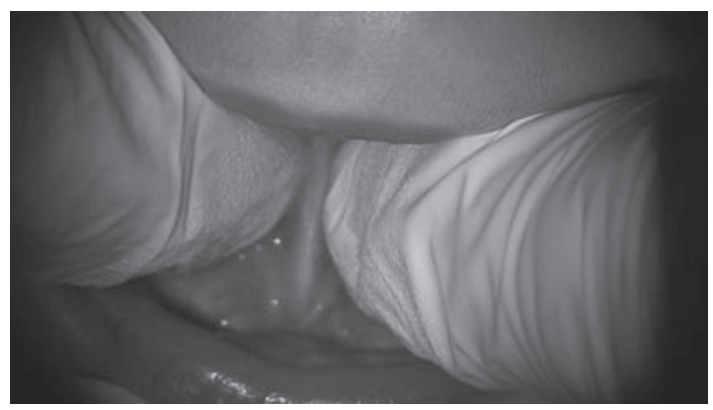

Class 4: 10-12 $\mathrm{mm}$ from tip of tongue

the lip-and tongue-ties resulted in significant improvement of the reflux symptoms. In this article, the author presents two cases where the infants were being treated for reflux symptoms without consideration or evaluation of the maxillary lip function: orankyloglossia.

\section{Case 1: Infant Breast- and Bottle-feeding}

The infant in Case 1 was born at 37 weeks gestation via cesarean section. Immediately after birth she experienced difficulty latching and feeding. At one month of age and during multiple office visits to her pediatrician, she presented with a significantly distended belly (see photo below).

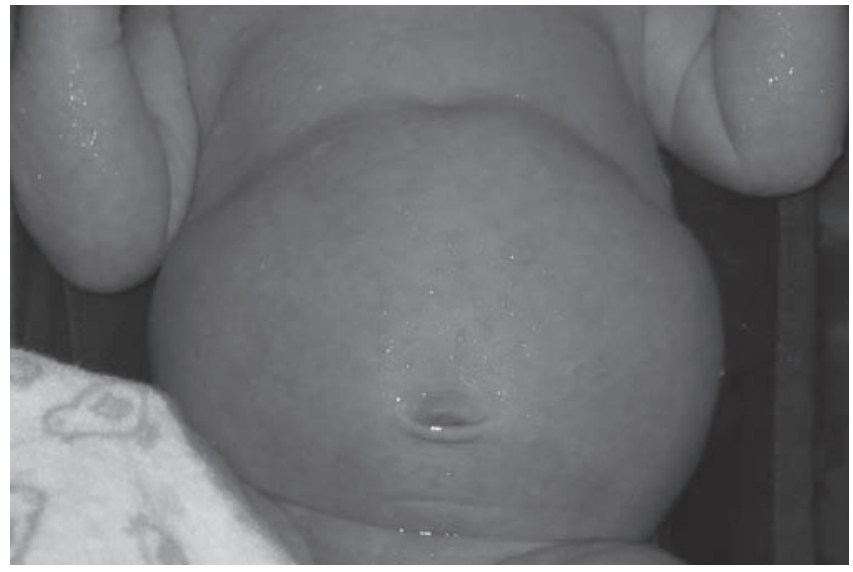

Aerophagia filling stomach

She developed reflux with projectile vomiting after feedings at approximately two months after her healthcare provider deemed breastfeeding to be inadequate. The pediatrician switched the infant from formula to formula five times with no improvement of symptoms. According to the infant's mother, the infant spent the majority of the day crying, arching her back, and clenching their hands in pain. The parents were told to be patient and that her infant would outgrow the problem by about three months of age.

At four months of age, she was placed on Prevacid (lansoprazole). After no (or little) relief of symptoms, the doctor added Miralax (polyethylene glycols) when the infant was six months old.

The symptoms remained and the infant was sent to see a neurologist, and then a developmental behavioral specialist, due to apparent delays in development. In an attempt to find the cause of the problems, the infant's medical work-up included genetic testing, metabolic assessments, and screening for RETT Syndrome and Crohn's Disease. At eight months of age, there was still no improvement in the acid reflux, and the infant was scheduled for barium swallow test to evaluate her gastrointestinal area.

The evening prior to the scheduled barium testing the patient had discussed her infant's problems with a relative who had an infant who had had similar symptoms. That parent previously had her infant's maxillary lip-tie and tongue-tie revised by the author. The relative suggested the infant might have both a lingual and maxillary tie, and referred her to the author's practice. The parent postponed the barium testing, called for an appointment, and was seen that morning. 


\section{Clinical Examination}

An oral examination revealed the infant had both a Class 4 maxillary lip-tie and an undiagnosed Class 3 tongue-tie. (See sidebars for Kotlow's clinical descriptions of tongue- and liptie classifications.)

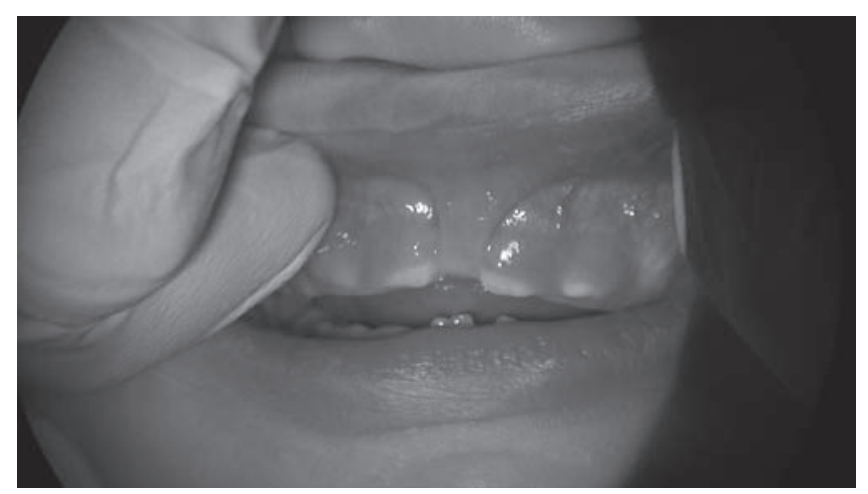

Class 4 Maxillary Lip-Tie

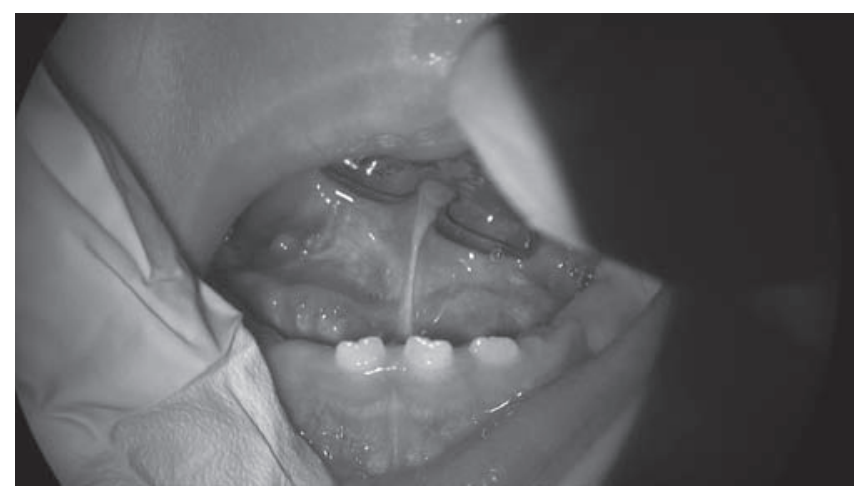

Class 3 Tongue-Tie

\section{Treatment}

Both the tongue-tie and lip-tie were revised in the dental office using local anesthesia (approximately $.25 \mathrm{cc}$ of $4 \%$ articaine 1:100,000 with epinephrine) and the Er: YAG laser (PowerLase AT @2940nm 20 HZ and 55 mj). (For more information on using lasers in newborns for soft-tissue surgery, go to www. kiddsteeth.com.)

\section{Post-surgery Follow-up Evaluation}

Twenty-four hours after the infant was treated, she experienced significant reduction in vomiting, gas, and crying. According to the parent:

After the laser surgery, we noticed immediate change in her feedings: quiet, more controlled, less spillage out of her mouth. She seemed to close better on the nipple. Her behavior and development improved. Within days she was making noises, smiling, laughing, and I noticed her hands less clenched and she was more interactive with her toys.
At one year of age, the infant had been off of the Prevacid (lansoprazole) and Miralax (polyethylene glycols), and her developmental milestones were appropriate for her age. The mother stated "prior to the surgery it was a scary eight months!"

\section{Case 2: Infant Bottle-feeding at Age 14 Weeks}

This particular case involved an infant who had other siblings being treated in the dental office for routine dental care. When the author was discussing the results of the other children's routine check-up dental visits, the mother brought up the problems her 14-week-old infant was experiencing. In this case, the infant's mother had had prior breast reduction surgery, which according to the mother, reduced her "lactation potential," resulting in the infant being exclusively bottle-fed breast-milk substitutes.

\section{Past Medical History}

The mother described the infant's following feeding scenario. Her infant experienced prolonged feeding, during which time the infant clicked (released or lost suction). This was followed by gagging, crying, and reflux. She was unable to hold a pacifier in her mouth, and had been placed on a milksubstitute formula because the pediatrician felt her crying and gas were due to formula allergy. The infant had been prescribed Prevacid (lansoprazole). However, the mother had not begun using it at this time.

\section{Clinical Oral Evaluation}

The clinical evaluation of the infant's maxillary frenum revealed a slight Class 2 lip-tie, and a significant anterior tongue-tie, inserting about $3 \mathrm{~mm}$ from the tip of the tongue into the base of the tongue (Class 4).

\section{Treatment}

The maxillary and lingual ties were revised using a $1064 \mathrm{~nm}$ semiconductor diode laser (Xlase pulsed at 200usec on and 100 usec off at $1.7 \mathrm{w}$ ), and a small amount of local anesthesia (approximately $.25 \mathrm{cc}$ of $4 \%$ articaine $1: 100,000$ with epinephrine).

\section{Post-surgical Evaluation}

Twenty-four hours after surgery, the mother indicated all symptoms were improving. After eight days, the mother indicated:

The infant no longer showed symptoms of gagging, vomiting, or reflux. The infant drained her bottle in 15 minutes and was able to burp herself. Her painful gas had resolved. The infant could now hold a pacifier. However, she was now a happy child and did not need a pacifier for comfort. 


\section{Conclusion}

Infants who experience "colic" or reflux symptoms may benefit from clinical oral examination and discussion with a qualified healthcare provider. The infant's oral examination may suggest a maxillary lip-tie and/or tongue-tie may be a contributing factor of an infant's inability to create an adequate seal. This may result in clicking and swallowing excessive amounts of air, which may result in reflux and coliclike symptoms. Revising the ties may result in a reduction or elimination of the symptoms (Buryk et al., 2011). Infants expressing these signs and symptoms should be evaluated for the possibility of abnormal frena attachments before extensive studies and medications are given to relieve the symptoms.

\section{References}

Buryk, M., Bloom, D., \& Shope, T. (2011). Efficacy of neonatal release of ankyloglossia: A randomized trial. Pediatrics, 128(2), 280-288.

Chitkara, D.K., Bredenoord, A.J., Wang, M., Rucker, M.J., \& Talley, N.J. (2005). Aerophagia in children: Characterization of a functional gastrointestinal disorder. Neurogastroenterololgy $\mathcal{E}$ Motility, 17(4), 518-522.
Courtiol, J. (2011). The cause and treatment of infant reflux. www.coliccalm.com/ baby_infant_newborn_articles/acid-reflux.htm

Fernando, C. (1998). Tongue-tie: From confusion to clarity. Sydney, Australia: Tandem Publications.

Kotlow, L. (2004a). Oral diagnosis of abnormal frenum attachments in neonates and infants: Evaluation and treatment of the maxillary and lingual frenum using the Erbium: YAG Laser. Journal of Pediatric Dental Care, 10(3), 11-14.

Kotlow, L. (2004b). Oral diagnosis of abnormal frenum attachments in neonates and infants. Journal of Pediatric Dental Care, 10(3), 26-28.

Kotlow, L. (2011). Diagnosis and treatment of ankylosis and ties maxillary fraenum in infants using Er:YAG and 1064 Diode lasers. European Archives of Pediatric Dentistry, 12(2), 106-112.

Kotlow, L. (2010). The influence of the maxillary frenum on the development and pattern of dental caries on anterior teeth in breastfeeding infants: Prevention, diagnosis and treatment. Journal of Human Lactation, 26(3), 304-308.

Li, R., Fein, S.B., Chen, J., \& Grummer-Strawn, L. (2008). Why mothers stop breastfeeding: Mothers' self-reported reasons for stopping during the 1 st year. Pediatrics, 122, 69-76.

Loening-Baucke, V. (2000). Aerophagia as causes of gaseous abdominal distention in a toddler. Journal of Pediatric Gastroenterology 8 Nutrition, 32(2), 204-207.

\section{Also from the Office of Women's Health Quick Data Online}

The 2011 Edition of the Women's Health and Mortality Chartbook is also now available. The Chartbook presents 28 health indicators, which highlight some of the key issues related to women's health that are being measured regularly at the state level. The Chartbook is a statistical resource on women's health in each of the states, the District of Columbia, Guam, Puerto Rico, and the U.S. Virgin Islands and was developed to provide readers with an easy-to-use collection of current state data on critical issues of relevance to women.

In addition, the 2010 Edition of the Health Disparities Profiles is now available. The Profiles present 21 health indicators that are being measured regularly at the state level and which highlight some of of the key areas related to health disparities among different populations. The Profiles is a statistical resource on population health for different racial and ethnic populations in each of the 50 states, the District of Columbia, Guam, Puerto Rico, and the U.S. Virgin Islands and was developed to provide readers with an easy-to-use collection of current state data on critical issues of relevance to population health. 